Accepted 2012 December 27 for publichtion in Astrophysical Journal

Preprint typeset using $\mathrm{LAT}_{\mathrm{E}} \mathrm{X}$ style emulateapj v. 12/16/11

\title{
NEARBY PLANETARY SYSTEMS AS LENSES DURING PREDICTED CLOSE PASSAGES TO BACKGROUND STARS
}

\author{
Rosanne Di Stefano ${ }^{1}$, James Matthews ${ }^{1,2}$, And SÉbastien Lépine ${ }^{3,4}$ \\ Accepted 2012 December 27 for publication in Astrophysical Journal
}

\begin{abstract}
The Einstein rings and proper motions of nearby stars tend to be large. Thus, every year some foreground stars within a few hundred parsecs of Earth induce gravitational lensing events in background stars. In some of these cases, the events may exhibit evidence of planets orbiting the nearby star. In fact, planets can even be discovered during relatively distant passages. Here, we study the lensing signatures associated with planets orbiting nearby high-proper-motion stars. We find the following. (1) Wide-orbit planets can be detected for all distances of closest approach between the foreground and background stars, potentially producing independent events long before and/or after the closest approach. (2) Close-orbit planets can be detected for intermediate distances of closest approach, producing quasiperiodic signatures that may occur days or weeks before and after the stellar-lens event. (3) Planets in the so-called "zone for resonant lensing" can significantly increase the magnification when the distance of closest approach is small, making the stellar-lens event easier to detect, while simultaneously providing evidence for planets. Because approaches close enough to allow planets to be detected can be predicted, we can plan observing strategies to take advantage of the theoretical framework built in this paper, which describes the sequence of expected effects in terms of a sequence of detection regimes.
\end{abstract}

Key words: planetary systems - stars; low-mass

\section{INTRODUCTION}

We are entering an era in which we can systematically predict close passages between nearby stars moving across the sky and background stars. Predictions are now possible because more than 2 million nearby stars with the largest motions have been identified (Lépine \& Shara 2005, Lépine \& Gaidos 2011), while deep surveys are mapping large background fields (Kaiser 2004; Aihara et al. 2011)

During the closest passages, the combined gravitational influence of the nearby star and its planets can produce distinctive lensing events which reveal the presence of the planets. In fact, even when the nearby star itself does not come close enough to the background star to produce detectable lensing effects, it should still be possible to probe for the presence of planets. In this paper we develop the theoretical framework needed to take full advantage of the planet-lens opportunities afforded by close passages between nearby and more distant stars.

For individual cases in which a close passages is predicted, we consider the possibility that the nearby star making the close passage has planets in orbits with semimajor axes ranging outward from about a tenth of an Einstein radius. For each orbital size, we predict the full range of detectable signatures as a function of time to the point of closest approach. In a future paper, based on Di Stefano, Matthews, \& Lépine (2012), we turn to

\footnotetext{
${ }^{1}$ Harvard-Smithsonian Center for Astrophysics, 60 Garden Street, Cambridge, MA 02138

${ }^{2}$ School of Physics \& Astronomy, University of Southampton, Southampton, SO17 1BJ, UK

${ }^{3}$ Department of Astrophysics, Division of Physical Sciences, American Museum of Natural History, Central Park West 79th Street, New York, NY 10024

${ }^{4}$ Department of Physics, Graduate Center, City University of New York, 365 Fifth Avenue, New York, NY 10016, USA
}

the design of observing programs that are optimized to detect the signatures predicted we predict here.

Section 2 provides a brief overview of the relevant lensing background. In $\S 3$ we also discuss other relevant characteristics of planetary systems. Of particular importance is the orbital period. Because stars making predicted passages will generally be nearby, their Einstein radii can be small. Thus, many of the planets that can be discovered through lensing may have short orbital periods. The change in orbital phase during the interval of close passage can increase the probability of planet detection. In addition, the reflexive orbital motion of the central star can effectively be magnified, providing another avenue toward the detection of planets. We also consider the possibility that the planets to be detected will lie in the habitable zone.

The types of lensing signatures associated with planets depend strongly on the orbital separation. It is convenient to divide the full range of separations into three subranges or zones: the close-orbit zone, the "resonant" zone, and the wide-orbit zone. Planets in each zone produce distinctive signatures. The signatures produced by planets in a given zone can, however, be detected only if the background source passes within a well-defined range of distances of closest approach. We therefore define, for each zone, a detection "regime" of distances of closest approach: when the source enters the regime associated with a particular "zone" of planetary positions, we have a significant chance of detecting any planets that may inhabit that particular zone around the nearby star.

By utilizing the notion of three separate lensing regimes, we can organize the effects according to their likely times of occurrence. We begin this process in Section 4 , where we consider the regimes in the order of increasing proximity to the central star, which is also 
the order in which planets can be detected: wide, close, and then resonant. In Section 5 we summarize our results and prepare the way for their use in real observing programs, which will be the subject of a future paper, based on Di Stefano, Matthews, \& Lépine (2012).

\section{BACKGROUND: FROM CLOSE PASSAGES TO LENSING EVENTS}

Given enough high-resolution images of a region, the relative motion of the stars it contains can be measured and close passages can be predicted. We are interested in the question of whether close passages will produce lensing events (Di Stefano 2008a, 2008b). The simplest case to consider is lensing of a distant background star by a star much closer in the foreground.

Gravitational lensing has both photometric and astrometric effects (e.g., Einstein 1936; Dominik \& Sahu 2000). One clear-cut astrometric effect is illustrated by the concept of the Einstein angle, $\theta_{E}$. The Einstein angle is defined to be the angular radius of the ring that would form the image of the source, were the source, lens, and observer to be perfectly aligned. In the absence of perfect alignment, a point lens produces two images. If $M_{*}$ is the mass of the lens, $D_{L}$ is the distance to the lens, and $D_{S}$ is the distance to the lensed source, then

$$
\theta_{E}=10 \operatorname{mas}\left[\left(\frac{M_{*}}{0.1 M_{\odot}}\right)\left(\frac{8 \mathrm{pc}}{D_{L}}\right)\left(1-\frac{D_{L}}{D_{S}}\right)\right]^{\frac{1}{2}}
$$

By comparing the size of the Einstein angle to the distance of closest approach, we can determine how large the effects of lensing will be. It is therefore important to be able able to estimate the value of $\theta_{E}$.

Prediction of close passages is most likely to be possible when the nearby star is a high-proper-motion star (HPMS), with proper motion $\mu$ larger than a few tens of milliarcseconds per year. Such stars are near enough to us that the value of $\sqrt{1-D_{L} / D_{S}}$ is likely to be close to unity. We can therefore often obtain a useful estimate of the value of $\theta_{E}$ if we can estimate the mass of the nearby star and its distance from us.

Because we are considering nearby stars with measured proper motions, in most cases we will know the star's spectral type and can use it to estimate the values of $D_{L}$ and $M_{*}$. For some nearby star we can do even better, because the geometric parallax is known and provides a high-precision measurement of $D_{L}$. VB 10 is one such well-studied star, with an estimated distance of $5.8 \mathrm{pc}$ and an estimated mass of $0.075 M_{\odot}$. The Einstein angle for VB 10 is approximately equal to 10 milliarcseconds (Lépine \& DiStefano 2012).

The astrometric effects of lensing fall off slowly with the angular separation between the source and lens, $u$. If $u$ is expressed in units of $\theta_{E}$, then in the limit of large $u$, the shift of the centroid is roughly equal to $\theta_{E} / u$. Prospective space-based missions, such as GAIA and Sim-Lite potentially could measure centroid shifts of tens to hundreds of microarcseconds, and may therefore be able to detect the effects of lensing event when the distance of closest approach is as large as an arcsecond or more. Measuring astrometric shifts provides the advantage of a direct measurement of $\theta_{E}$. If, therefore, $D_{L}$ is well measured, the gravitational mass can be measured to high precision. Such a direct, high-precision measurement can have important scientific relevance. For example, the estimated mass of VB 10 lies close to the boundary between stars and brown dwarfs, so that a mass measurement would provide a particularly valuable data point. Other nearby objects for which direct mass measurements are potentially important include brown dwarfs and white dwarfs.

In this paper we will concentrate on the photometric effect of lensing, i.e., the magnification of the background source. A unique value of the magnification is associated with each value of $u ; A=A(u)$. The fractional magnification, $A-1$, is roughly 0.34 for $u=1,0.06$ for $u=2$, and 0.01 for $u=3.5$. For large $u$, the magnification falls off as $1 / u^{4}$. Thus, if, for example, an event with a peak magnification of $2 \%$ can be reliably identified, then the required angle of closest approach is $\sim 3 \times \theta_{E}$; if passages this close can be predicted, then we can be assured of detecting lensing of the background star by the foreground star.

A lensing model fit to the magnification light curve allows us to derive the value of the Einstein diameter crossing time, $\tau_{E}=2 \theta_{E} / \mu$, where $\mu$ is the proper motion. The value of $\mu$ can be measured for nearby stars, as it has been for VB 10. With $\tau_{E}$ measured from the light curve, the value of $\theta_{E}$ can be determined from the event itself, without the need for astrometric measurements.

\section{THE EFFECTS OF PLANETS}

Because we are interested in discovering planets orbiting the nearby star, the orbital separation between the planet and its star, generally expressed in units of the star's Einstein radius, will play a key role. It is therefore important to introduce the Einstein radius, $R_{E}=D_{L} \theta_{E}$.

$$
R_{E}=0.08 \mathrm{AU}\left[\left(\frac{M_{*}}{0.1 M_{\odot}}\right)\left(\frac{D_{L}}{8 \mathrm{pc}}\right)\left(1-\frac{D_{L}}{D_{S}}\right)\right]^{\frac{1}{2}}
$$

If $a$ is the instantaneous projected separation between a nearby star and a specific planet it may harbor, then $\alpha=a / R_{E}$ is the quantity that determines the types of light curve features that can provide evidence that the planet exists. It is therefore useful to delineate certain "zones" around the stellar lens, each zone defined by a range of values of $\alpha$.

\subsection{Orbital Periods}

Equation (2) demonstrates that, for planets that are close to us, the Einstein radius, $R_{E}=D_{L} \theta_{E}$, can be relatively small 5 The exact value of $R_{E}$ depends on both the lens mass and on the value of $D_{L}$. Nevertheless, for a wide range of stellar masses and distances to the lens (within a few hundred pc), $R_{E}$ can be smaller than an AU.

For a face-on circular orbit, the value of $\alpha$ is constant

\footnotetext{
5 The Einstein radius scales as $D_{L}^{\frac{1}{2}}$ for nearby lenses, while the size of the Einstein ring, $\theta_{E}$, scales as $D_{L}^{-\frac{1}{2}}$ for nearby lenses
} 
and is equal to the semimajor axis. In this case,

$P_{\text {orb }}=(26.1$ days $) \alpha^{\frac{3}{2}}\left(\frac{M_{*}}{0.1 M_{\odot}}\right)^{\frac{1}{4}}\left(\frac{D_{L}}{8 \mathrm{pc}}\right)^{\frac{3}{4}}\left(1-\frac{D_{L}}{D_{S}}\right)^{\frac{1}{4}}$

In general, the orbit will not be face-on, and may also be elliptical. Thus, $\alpha=\alpha(t)$, and the semimajor axis is $p(t) \alpha(t)$, where the instantaneous value of $p(t)$ can be larger than or smaller than unity, depending on the orbital inclination, eccentricity, and phase. For the purpose of considering specific well-defined cases, we will use circular face-on orbits as examples. We note however, that when deriving models to fit observed light curves, the full range of possible orbits must be considered.

Short orbital periods mean that the orbital phase can change significantly during the interval when the lens is close to the source. For example, if $R_{E}=0.08 \mathrm{AU}$, then the time taken by a $10 \mathrm{~km} \mathrm{~s}^{-1}$ lens to move through a distance of $2 R_{E}$ is 27.7 days. Thus, for a lens with $M_{*}=$ $0.1 M_{\odot}$, located 8 pc away, a full orbital period can occur (if the orbit is circular and face-on, with $\alpha=1$ ) during the time required by the lens to move across an Einstein diameter. More revolutions can occur if $\alpha$ is smaller. In fact, because the ratio of $P_{\text {orb }}$ to $R_{E}$ is proportional to $D_{L}^{\frac{1}{4}}$, and to $M_{*}^{-\frac{1}{4}}$, there are wide ranges of parameters for which orbital motion is important.

It is therefore more likely that regions in which the isomagnification contours are perturbed from the pointlens form will pass in front of the source, increasing the probability of planet detection. In some cases, the region in the lens plane with magnification deviations can pass over the source star more than once. The repetition of deviations makes them more likely to be detected and correctly identified.

Overall, the probability of planet detection can be significantly larger for HPMSs than it is for lens stars located several kpc away, the typical location for the lenses discovered by the lensing teams [e.g., the OGLE (Udalski 2003) and MOA (Bond et al. 2001) teams, both of which are presently active].

\subsection{Magnification of Reflexive Motion}

As a planet orbits a star, the star wobbles in its own smaller orbit. When the star serves as a lens, small changes in its position can make a measurable difference in the magnification it produces in a background star. The magnification enhances the effects of stellar wobble, making it more readily detectable.

In units of the Einstein angle, the wobble of the star has amplitude

$$
\delta=\frac{m_{p}}{M_{J}} \alpha \times f
$$

where $M_{J}$ is the mass of Jupiter, and the value of $f$ depends on how much of the orbit is executed during the time when the magnification is detectable; if a full orbit is executed $f$ could be as large as about 2 , or much smaller, if the shift in orbital phase during the event is small.

Whether the stellar wobble is large enough to produce a detectable change in magnification depends on how close on the sky the source star is to the lens. Let $\beta$ represent the projected distance between source and lens, expressed in units of the Einstein radius. The magnitude variation can be expressed as $(A(\beta-\delta)-A(\beta+\delta))$. The value of $\delta$ will generally be smaller than $\theta_{E}$. For close approaches, where the reflexive motion is most magnified, the value of $\beta$ is also small. Thus, the magnitude of the fractional change, $\Delta A / A$ in magnification can be written as follows.

$$
\frac{\Delta A}{A}=\left|\frac{\beta \delta}{\beta^{2}-\delta^{2}}\right|
$$

For $\delta$ smaller than $\beta$, we may have $\Delta A / A=\delta / \beta$. Thus, $\Delta A / A$ may well correspond to a measurable change in the magnification. For close orbits (small values of $\alpha$ ), this change will be small, but it will be periodic. For large $\alpha$, the change may be large, but it will not repeat. Instead, it can be measured by a shift in the peak magnification relative to the baseline.

\subsection{The Habitable Zone}

The planets many astronomers are most eager to discover are those that could harbor life. It is also desirable that lensing discovers planets that could be habitable (Di Stefano 1999). Di Stefano \& Night (2008) pointed out that, when the lens is a nearby dwarf star, planets that can produce detectable lensing signatures are more likely to lie in the zone of habitability. This should apply to the HPMSs for which we can predict events.

The zone for habitability is a concept that is based on the premise that a planet orbiting a star is more likely to harbor life when its surface temperature is such that it can support liquid water. This will be the case, when the flux incident on the planet from the star is comparable to the flux received by the Earth from the Sun. This condition on the flux is satisfied if

$$
D_{L}\left(1-\frac{D_{L}}{D_{S}}\right)=\frac{125 \mathrm{pc}}{\alpha^{2}}\left(\frac{M}{M_{\odot}}\right)^{2.5}
$$

The habitable zone is an annulus that is large enough that some systems may have values of $D_{L}$ and $M_{*}$ roughly compatible with the habitable zone for a broad range of $\alpha$ values. In this case, one close passage might be able to discover multiple planets that are potentially habitable.

\section{ZONES AND DETECTION REGIMES}

The characteristic signatures associated with planets are different for different orbital separations between the planet and the star. It is therefore convenient to define three distinct zones in which planets may be located: the close-orbit zone, the resonant-orbit zone, and the wideorbit zone. Each zone is defined by a range of values of $\alpha=a / R_{E}$, representing the instantaneous value of the projected orbital separation between the planet and its star, expressed in units of the Einstein radius.

In order to detect planets located in a given zone, the source must pass through a region in which there are planet-produced perturbations in the magnification pattern. For planets in each zone, there is an associated region where the deviations are most noticeable. The source track must pass behind this perturbed region in order for the presence of the planet to be detected. We call this region the detection regime associated with the 


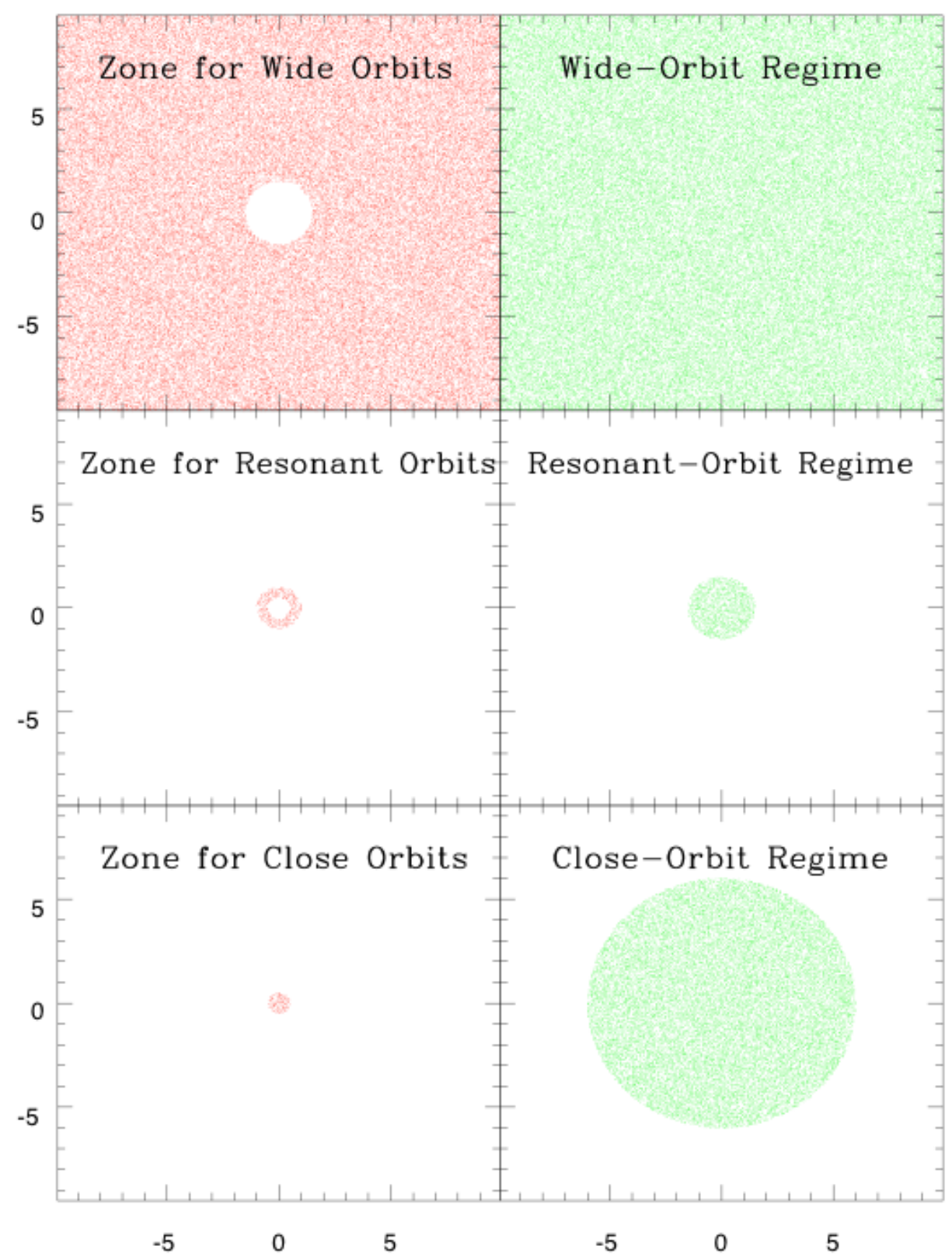

Figure 1. Panels on the left show the zones within which planets can be located. Bottom panel: planets in the close-orbit zone have $\alpha<0.5$. Middle panel: planets in the zone for resonant lensing have $0.5<\alpha<2$. Top panel: planets in the wide-orbit zone have $\alpha>2$. Panels on the right show the corresponding detection regime. In each case, the detection regime contains the zone where the planets are located, but also a larger region. Bottom panel: The close-orbit detection regime extends to just over $\frac{1}{\alpha_{\min }}-\alpha_{\min }$, where $\alpha_{\text {min }}$ is the minimum value of $\alpha$ for which the planet-induced deviations are detectable. Here we have taken $\alpha_{\min } \sim 0.15$. Middle Panel: The detection regime for resonant-orbit planet includes the resonant zone itself and is augmented by a disk around the stellar position. Top Panel: The wide-orbit detection regime extends from the origin to the widest planet in the planetary system, which can be at a distance of hundreds of Einstein radii.

planet-containing zone. The detection regime is defined by the range of distances of closest approach compatible with event detection. As in $\S 3$, it is convenient to let $\beta$ represent the distance of closest approach, expressed in units of $R_{E}$.

As we will see, the detection regime for each zone contains the zone, but is larger. The zones and their associated regimes are described below and are illustrated in Figure 1. We note that the boundaries between the zones containing each category of planetary orbits are fuzzy, in the sense that there are generally not abrupt change in the signatures at specific values of the projected orbital separation, and the signatures also depend on the mass ratio. Nevertheless, the signatures change in a significant and systematic way as $\alpha$ increases, making it useful to delineate the three zones we discuss below, and the associated detection regime associated with each.

\subsection{Close-Orbit Planets}

A point lens produces isomagnification contours that are circles centered at the position of the lens. The addition of a second mass changes the isomagnification contours in a way that can be highly non-linear. In addition, caustic curves are introduced. When the track 


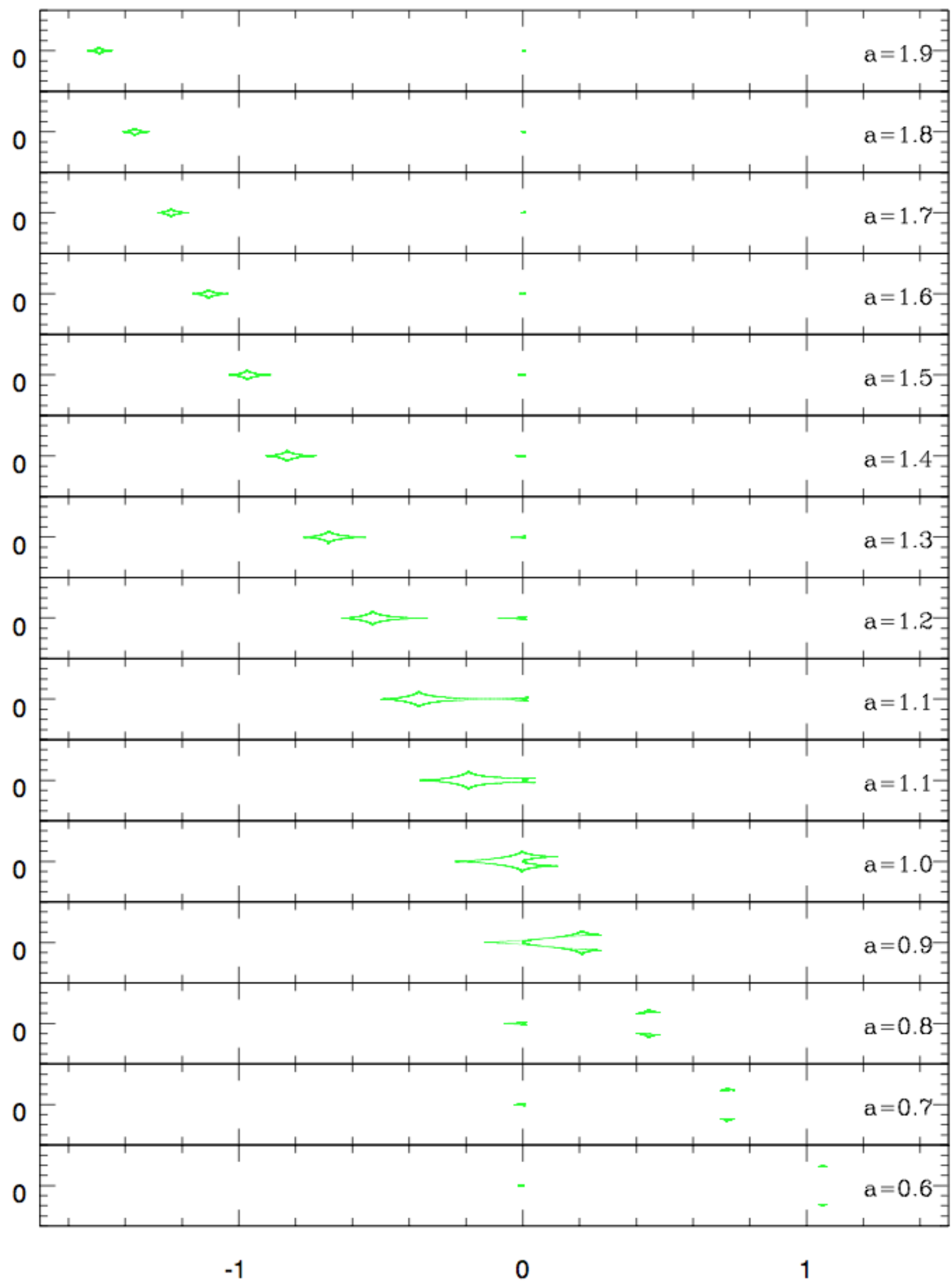

Figure 2. Caustic structures for a sequence of values of $\alpha: 0.6-1.9$ In each case $q=0.001$. 
of the source passes behind a caustic curve, the number of images switches from three (five) to five (three); if the source is a point, the magnification becomes infinite at the time of crossing. The positions and sizes of the caustics are illustrated in Figure 2 (see also Wambsganss 1997); the planet used to compute the structures shown in Figure 1 had a mass equal to 0.001 that of the central star. The orbital separation in the bottom panel is $\alpha=0.6$. In this bottom panel we see that the caustic structures are small, so that it is unlikely that any given source track will encounter one or more of them. Furthermore, two of the structures are located more than an Einstein radius from the central star, in fact much farther from the star than is the planet itself.

Here we will consider close-orbit planets that represent the small- $\alpha$ continuation of Figure 2. We will call a planet a close-orbit planet if $\alpha<0.5$. This zone is shown in the left-bottom panel of Figure 1. Interestingly enough, the detection regime for close-orbit planets is much larger than the limited zone they occupy. This is illustrated in the right-bottom panel of Figure 1, and is explained below.

As the value of $\alpha$ decreases, the trend illustrated in Figure 2 continues. The caustics move out to larger distances from the star. They can be found in a small annulus centered on $R_{\alpha}=\frac{1}{\alpha}-\alpha$. The caustics themselves are very small, and it is unlikely that the source track will intersect them. There is, however, a more extended region around the caustics where the magnification is distorted from the point-lens form. The size of this region is comparable to but somewhat larger than the distance between the caustics, which increases as $\alpha$ decreases. Isomagnification contours from closer in are pulled out, and others from further out are pulled in. This means that, for $\alpha \sim(0.2-0.3)$, for example, there can be deviations of a few percent that start when the source is somewhere between $4.8 R_{E}$ and $2.7 R_{E}$. These are detectable for hours to days, and can repeat as the perturbed region is whipped around to follow the motion of the close-orbit planet.

Thus, the outer portion of the close-orbit detection regime starts at slightly larger values of $u$ than $R_{\alpha}$. It generally extends inward to the origin, since the reflexive motion of the lens star will repeat, making it possible to detect the periodicity and to infer the presence of the planet. The discussion above demonstrates that, for close-orbit planets, the detection regime is significantly larger than the zone within which the planets lie. details can be found in DiStefano(2012). Note again that the distinction between the zone and the regime is that the former contains the planets, while the latter is the region through which the source must pass if we are to detect evidence of the planets, as is illustrated in Figure 1.

\subsection{Resonant-Orbit Planets}

The panels shown in Figure 2 cover the ranges of separations from just over $0.5 R_{E}$ to just under $2.0 R_{E}$. Planets located in this annulus are said to be in the zone for resonant lensing (Mao \& Pacyński 1991; Gould \& Loeb 1992). When the planets lie in the zone for resonant lensing, evidence for planets comes from light curves in which the source tracks pass near or behind the caustics, producing a significant deviation from the point-lens form. It is worth noting that the terms "resonant-orbit" or "zone for resonant lensing" do not refer to resonance in the dynamical sense. Instead, the term derives from the circumstance that discoveries of planets in this zone tend to be associated with caustic crossings which can produce large magnifications that rise and fall over very small time intervals.

The regime through which the source must pass in order to detect evidence of planets residing in the zone for resonant lensing extends from the origin to about $2 R_{E}$. Thus, like the close-orbit regime, the resonant regime is larger than the zone within which the planets reside. The additional area is simply the relatively small area of the disk that extends from the origin to $0.5 R_{E}$. Although small, this region contains a caustic structure that is associated with the central star. Thus, the track of any source that passes very close to the star crosses a caustic and can therefore exhibit evidence of the planet's presence (Griest \& Safizadeh 1998).

\subsection{Wide-Orbit Planets}

If the planet lies in at larger values of $\alpha,(\alpha$ greater than about 2), we will refer to it as a wide-orbit planet. Wideorbit planets can serve as almost independent lenses, and they can also be detected through deviations in the light curve associated with lensing by the central star (Di Stefano \& Scalzo 1999a, 1999b).

Wide orbit planets can be detected even for distant approaches, i.e., for large values of $\beta$. The reason is simply that dramatic events can be created when the source path comes close to the Einstein ring of the planet, which is, by definition, far from the central star. Such events can occur at times much earlier than or later than the time of closest approach between the foreground and background star. Thus, the wide-orbit detection zone has an outer edge that is as wide as the widest orbit in the planetary system serving as lens. In addition, evidence of wide-orbit planets can be detected during close approaches between the foreground and background star, since the presence of a wide planet can affect the shape of the stellar-lens light curve. Thus, the wide-orbit detection regime extends inward to the origin. For any given wide-orbit planet, the detection regime may be viewed as a large-radius annulus, centered on the planet's position, combined with a disk, centered on the lens star. In fact, however, because a given planetary system may host a sequence of potentially-detectable wide-orbit planets, it makes sense to view the detection regime as an extended region, as shown in the upper right panel of Figure 1.

\begin{tabular}{|c|c||c|c|}
\hline Regime & $\beta$ & $\alpha$ & Light Curve \\
\hline Resonant & $\lesssim 2.0$ & $0.5-2.0$ & Figure 7 \\
Close & $\lesssim(5.0-6.0)$ & $\lesssim 0.5$ & Figures 5 and 6 \\
Wide & all & $\gtrsim 2.0$ & Figure 4 \\
\hline
\end{tabular}

Table 1

A summary of the properties of the three different zones (defined by $\alpha$ ) and regimes (defined by $\beta$ ). $\alpha$ is the orbital separation in units of $\theta_{E}$ and $\beta$ is the distance of closest approach in units of $\theta_{E}$. See Figure 1. The size of the outer boundary of the close-orbit regime is determined by the magnitude of the smallest deviations which are detectable, and can be larger or smaller than $(5-6) R_{E}$. 


\section{FOLLOWING A SOURCE STAR THROUGH THE THREE DETECTION REGIMES}

When the foreground star is nearby, its proper motion is the primary source of relative motion between it and the background star. For the purposes of this discussion, however, we will work in a frame in which the foreground star is at rest, and consider the motion of the background source. We also note that, if the foreground star is close enough to us, geometric parallax introduces a measurable curvature into the relative paths. For the purposes of this discussion we will ignore this effect. When, however, we consider a specific nearby lens, such as VB 10, which is only 5.8 pc away, parallax must be explicitly included.

As the source moves toward the foreground star, it first enters the regime for the detection of wide-orbit planets. If the position of one or more wide planet happens to be favorable for detection, because the source will pass close to it, planet-lens events can occur long before the source passes close to the foreground star. In fact, even if the approach between the two stars is never close enough that the foreground star lenses the background star in a detectable way, it may be possible to detect wideorbit planets. Thus, the wide-orbit regime is explored for many planetary systems, even if $\beta>>1$.

As the source moves closer and closer to the foreground star, planets in orbits that are "wide" but with shorter orbital periods can also serve as lenses. The orbital motion associated with the shorter orbital periods increases the probability of an event with a wide-orbit planet.

If $\beta$ is smaller than about 5 or 6 , the source star enters into the regime for the detection of close-orbit planet:6. In this regime, the probability of detecting the presence of a close-orbit planet approaches unity. In addition the probability of detecting wide-orbit planets increases. Thus, even if the value of $\beta$ never reaches a value as small as 2 , it is possible to detect planets in a wide range of orbits, from the widest $(\alpha>2)$ to the closest $(\alpha<0.5)$.

Finally, if $\beta<2$, the source enters the resonant-orbit planet detection regime. While the orbital periods of resonant-orbit planets are longer than those of close-orbit planets, they can nevertheless be short enough to significantly increase the probability of planet detection. In the region $\beta<2$, planets in all three zones can be detected.

The parameters specifying the three zones and regimes are summarized in Table 1. In the next section we imagine following the track of the source from the outer edges of the wide-orbit regime to a location very close to the foreground star.

\subsection{Wide-Orbit Planets}

\subsubsection{Magnification Patterns for Wide-Orbit Planets}

A star and planet can each produce well-separated events for projected separations as small as $1.5 \theta_{E}$, although this result depends on the value of the mass ratio and on the photometric sensitivity of the observations. In

6 The outer radius of the close-orbit regime roughly coincides with $R_{\alpha}=\frac{1}{\alpha}-\alpha$, and therefore extends out to values of $\alpha$ associated with the innermost stable orbit, which generally has $\alpha<<0.1$. For very small values of $\alpha$, however, there are detectability issues, since the deviation of the planet-induced perturbation from baseline is well under a percent. Here we have somewhat arbitrarily taken the outer edge of the close-orbit regime to have $R \sim 5-6$, but note that the most appropriate choice depends on the size of the light-curve effects that can be reliably detected. this paper, we consider the inner boundary of the wideplanet zone to be approximately $2 \theta_{E}$.

The wider the planetary orbit, the less likely it is that the track of the source will pass behind both the planet and the central star (see Di Stefano \& Scalzo 1999b for probability estimates). Nevertheless, even when only the planet-lens event occurs, the shape of the light curve associated with lensing by the planet can be influenced by the presence of the star until the value of $\alpha$ is fairly large. Figure 3, for example, shows that the $1 \%$ isomagnification contour is significantly distorted from a circular form, even for $\alpha \sim 6$. The significance of these distortions is twofold: they influence the light curve characteristics and, because they increase the linear dimensions of the region within which the magnification is significant, they increase the event probability.

Similarly, the isomagnification contours associated with the star can be perturbed by the presence of a wideorbit planet. The stellar-lens event may therefore show evidence of the planet, even in cases in which the planet does not produce an independent event. This is why the wide-orbit detection regime extends inward to the origin. In other words, the entire lens plane, from the origin out to the largest expected planetary orbit, comprises the wide-orbit detection regime.

\subsubsection{Independent Events by Wide-Orbit Planets}

Consider a wide-orbit planet with projected orbital separation $\alpha>2$. Let the distance of closest approach, which will take place at time $t_{0}$, be equal to $\beta$. The path of the source will intersect a circular orbit of radius $\alpha \theta_{E}$ at two times, given by the following equation.

$$
\left|t-t_{0}\right|=100 \text { days }\left(\frac{0.01 \frac{\theta_{E}}{\text { day }}}{\mu}\right)\left(\alpha^{2}-\beta^{2}\right)^{\frac{1}{2}}
$$

Thus, at approximately these two times it is possible for a wide-orbit planet in a circular orbit of radius $\alpha$ to produce a lensing event. The event would last for a time roughly proportional the square root of the planet mass $7^{7}$ For $\alpha>>2$ the light curve would have characteristics like those expected from an isolated lens. The closer the value of $\alpha$ to the inner edge of the wide-orbit zone, the more perturbed would be the light curve from the standard point-lens form.

Note that the independent planet-lens event can happen many days before or after the time of closes passage between the foreground and background stars. Equation 7 highlights an important feature of predicted events, or indeed any event in which the angle of closest approach is known. During any given day before or after the event, we can compute the specific value of $\alpha$, the projected orbital separation, of the planet whose lensing signature we can detect on that day. This is so whenever we know the HPMS's proper motion $\mu$, and can estimate the values of $\beta$ and $t_{0}$. Actually, given $\mu, \beta$, and $t_{0}$, we can compute a small range of possible values of $\alpha$, since the size of the lensing region around the planet has a finite extent which can be computed for each set of values of $q$ and $\alpha$ (Figure $3)$. Uncertainties in the path also produce uncertainties

7 The planetary Einstein radius is smaller than that of the star by a factor of $\sqrt{M_{P} / M_{*}}$, where $M_{p}$ is the mass of the planet. 


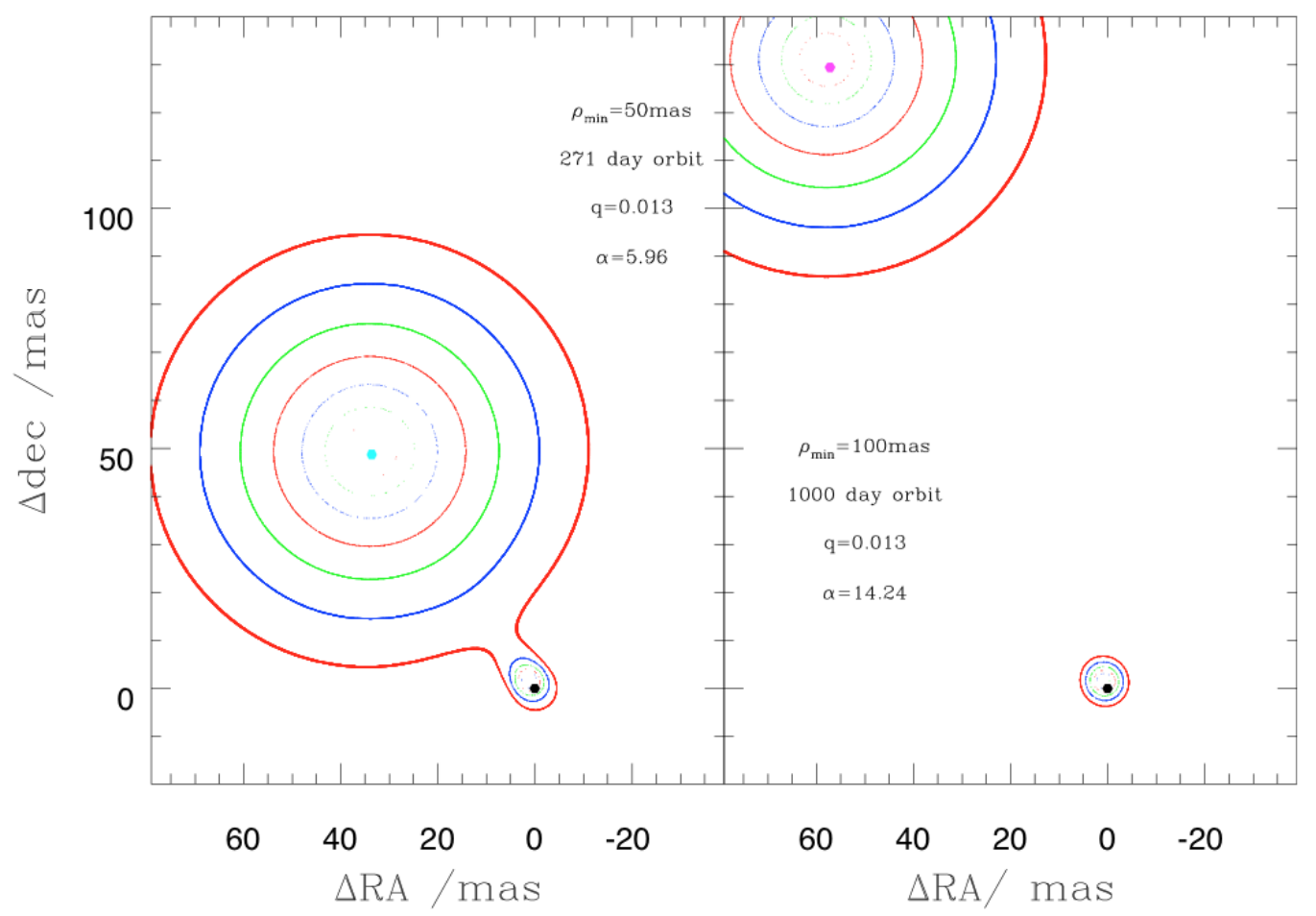

Figure 3. Isomagnification contours for two different wide orbits, where $\Delta R A=R A-R A_{\text {source }}$ and $\Delta d e c=d e c-d e c_{\text {source }}$ Left: $\alpha=5.96$, corresponding to a 271 day orbit, and Right: $\alpha=14.2$, corresponding to a 1000 day orbit. We show each system at a time when the position on the sky of both the planet and the source star is $(0,0)$. The planet produces an independent event by lensing the source star. The isomagnification contours associated with the planet can be clearly identified, and the perturbation in both the position and size of the contours can be seen. The outer red contour corresponds to a magnification $(A-1)=0.01$, and the magnification associated with each contour increases by a one-magnitude increment (a factor of $\sim 2.5$ ) as they get closer to the lens. (Note that the isomagnification contours associated with the planet are significantly influenced by the central star for $\alpha=5.96$; for the wider orbit the effect of central star on the region around the planet is smaller.

in the possible day-by-day values of $\alpha$. Thus, if an event is detected on a specific day, say the 30th day after the main event, we can immediately estimate the value of $\alpha$. The features of the associated light curve, including the value of $\tau_{E}$, allow us to estimate the mass of the planet because the proper motion can be well-measured.

Conversely, if no detection is made on a given day, we know what type of orbit is not producing an event. Lack of evidence for an event on any given day does not translate to evidence for lack of a planet. It does, however, allow us to place limits on the possible presence of a planet as follows. We consider the times the observations that have been made and, for each, the minimum value of the magnification to which the observation would have been sensitive. Then, by generating a large number of orbits for each value of $\alpha$ and each value of $q$, including a range of orbital eccentricities and inclinations, we can compute what fraction of the time a planet of each type would be detected. If the observations would have detected the planet $100 \%$ of the time, then the failure to observe the planet means that it is not there. If, however, the probability of detecting the planet is $\mathcal{P}$, then we can say that there is now a probability of only $1-\mathcal{P}$ that such a planet orbits the lens star. This numerical approach can be very accurate, but general planning can be facilitated even by less accurate analytic estimates.

A simple analytic approach to computing the probability that a wide-orbit planet will produce a separate short-duration event is to compare the size of the region within which a planet produces significant effects with the size of the orbit. This ignores velocity effects, but can nevertheless provide a reasonable estimate of the fraction, $\mathcal{P}$, of initial phases likely to produce an isolated planet-lens event.

To compute this fraction, we simply need to compute the ratio between (1) the size of the region around the planet that can produce detectable lensing effects, and, (2) the size of the orbit. We will express each in units of the Einstein radius. The denominator, the size of the orbit, is simply $2 \pi \alpha$. Developing a good approximation for the value of the numerator requires some care. First, the size of the region within which lensing can be detected depends on the sensitivity of the observations. If only magnifications of $34 \%(6 \%, 1 \%)$ or more are detectable, then the width of the lensing region is $1 \times\left(2 R_{E}\right)\left[2 \times\left(2 R_{E}\right), 3.5 \times\left(2 R_{E}\right)\right]$. The factor to the left in each case corresponds to the distance, in Einstein radii, at which the magnification becomes detectable. We have taken this into account by introducing a factor $F / 2$, which is unity when deviations of $6 \%$ are detectable, 0.5 if deviations must be larger than $34 \%$ are detectable, $3.5 / 2=1.75$ if deviations must be larger than only $1 \%$ to be detectable.

A second factor influences the size of the lensing region associated with the planet. This is the size of the orbit. For values of $\alpha$ in the range $2-6$, the lensing region is significantly stretched, with the amount of stretching also dependent on the mass ratio, $q$. For larger values of $\alpha$ the stretching is minimal. To take this into account, we have introduced the factor $E\left(q_{i}, \alpha\right) / 1.5$, which has the 


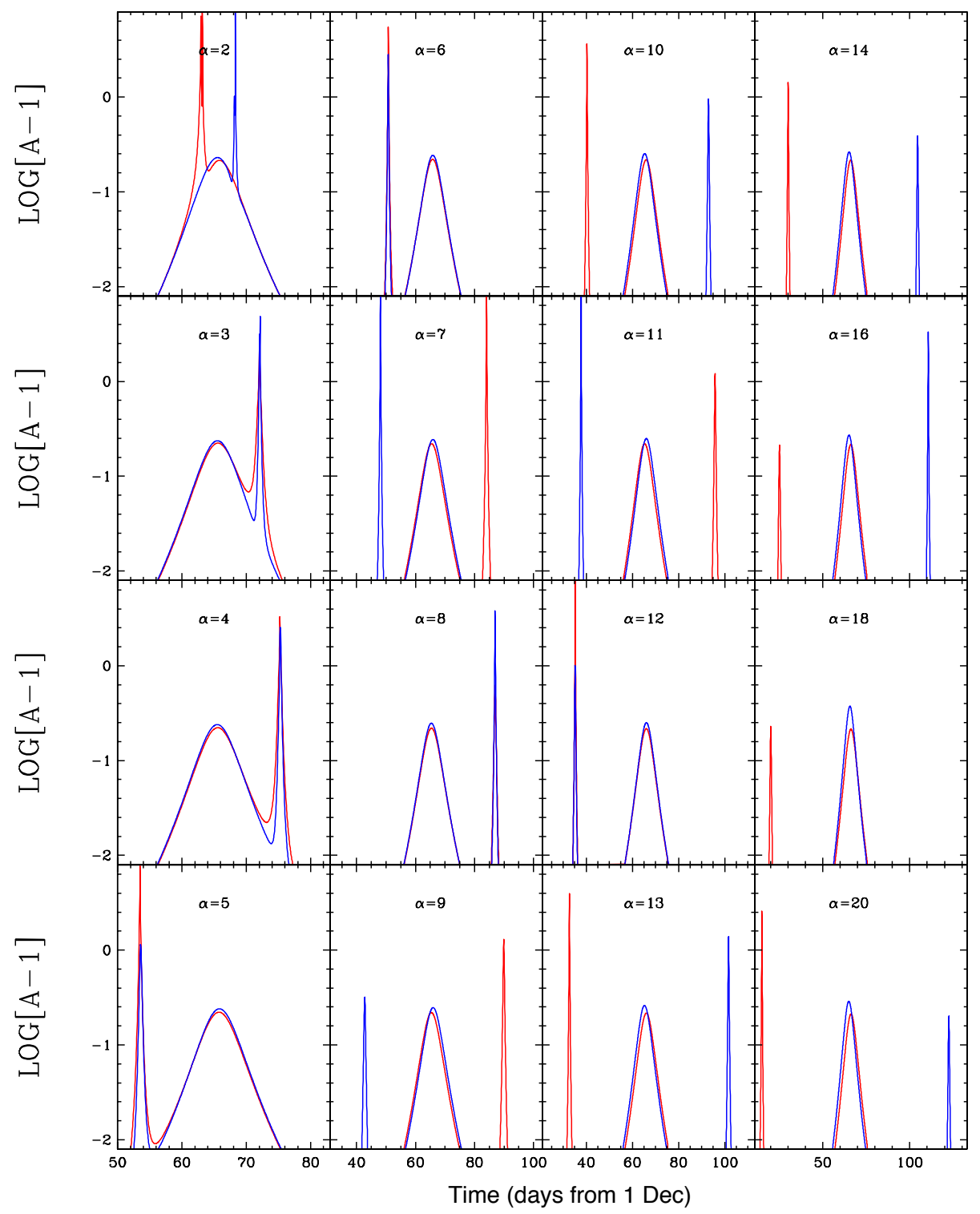

Figure 4. Wide orbit planets, $\beta=1$. Simulated lensing light curves for a variety of orbital separations for a stellar lens orbited by a Jupiter-mass planet. Red corresponds to clockwise orbits and blue corresponds to counterclockwise. The wider the orbit, the larger the time between the short-duration planet-lens event and the more perturbed the stellar-lens event. This supports the hypothesis that planetary events can be seen over a wide range of dates depending on the orbital separation, up to several months before and after the primary (stellar) event. The date is an arbitrary time prior to the time of closest passage.

value roughly equal to 1 for $\alpha=3$ and $q=0.001$.

With these definitions, the probability, $\mathcal{P}$, that a wideorbit planet will produce an isolated event is

$$
\mathcal{P}=0.36 \frac{F}{2} \sum_{i=1}^{n}\left(\frac{q_{i}}{0.01}\right)^{\frac{1}{2}}\left(\frac{1}{\alpha_{i}}\right)\left(\frac{E\left(q_{i}, \alpha_{i}\right)}{1.5}\right)
$$

where the sum is over wide-orbit planets. For $\alpha=3$, the contribution is 0.12 . A more accurate estimate of the probability would include the effects of orbital motion and parallax.

\subsubsection{Detecting evidence of the planet during the stellar-lens event}

Fits to light curves produced by the star have the potential to identify deviations from the point-lens form and to discover wide-orbit planets. This is the case even when the planet itself does not produce a separate de- tectable event.

The influence of the planet could be manifest through a slight distortion of the isomagnification contours surrounding the star. Such effects are likely to be most prominent in the low-magnification portion of the light curve.

The planet could also be detected through the reflexive motion of the star. As shown in Equation 4, the magnitude of the positional shift is small for small values of $\alpha$. Fortunately, for small $\alpha$, direct detection of the planet is more likely (Equation 8). Also for small $\alpha$, if the relative speed is not too high, the phase could change significantly enough during a close approach to affect the light curve shape. The wider the orbit, the larger the reflexive motion, and the more likely it is to produce deviations in the stellar-lens light curve from the pure point-lens form. This is illustrated in Figure 4. 


\subsection{Close-Orbit Planets}

If the foreground star happens to have planets with orbits much smaller than the Einstein radius, the lensing signatures can be significantly perturbed from the pointlens form. This is because there is a small region within which the isomagnification contours are distorted from the circular shape they would have had in the absence of the planet. These perturbations are maximized at a distance $R_{\alpha}=\left(\frac{1}{\alpha}-\alpha\right)>1.5$ from the center of mass. In the absence of orbital motion, there would be only a small probability that the distorted region would pass in front of the source. The orbital period is short for small values of $\alpha$, however, especially when the planetary system serving as a lens is nearby. Thus, even though the deviations tend to be small, repetition confers an advantage.

The light curves associated with close-orbit planets have the same overall shape as light curves produced by the central star. To detect evidence of the planets, we must be sensitive to small deviations of the type studied in Di Stefano (2012). The deviations have a characteristic up-down-up-down shape, and for nearby lenses, will generally repeat. Figures 3 through 6 of Di Stefano 2012 illustrate the deviations in the isomagnification contours and light curves for a "hot Jupiter", and for a Neptunemass and an Earth-mass planet located in the habitable zone of a dwarf star. In Figures 5 and 6 of this paper, we show the light curves for a variety of planets and planetary orbits, plotting the deviations, $A_{p l}-A_{p t}$, from the point-lens light curve produced by the central star alone. The specific foreground star for which the calculations were done is VB 10; the central star is, therefore a dwarf star of mass $0.075 M_{\odot}$. The value of $R_{E}$ is roughly 0.08 AU. Here we explain the patterns.

The most distinctive features are repeating deviations that start in the wings (i.e., the low-magnification portion) of the light curve. For each value of $\alpha$, the deviations start as the source star approaches a distance $R_{\alpha}$ from the central star. The deviations are larger for larger values of $\alpha$. In fact, if the observations are able to reliably detect deviations of size $\delta$, then close orbit planets with semimajor axes in the range between $\alpha_{\text {small }}$ and 0.5 can be detected, where

$$
\alpha_{\text {small }}=0.84 \delta^{\frac{1}{4}} \text {. }
$$

This effect is illustrated in the early-time and late-time deviations seen in the light curves of Figures 5 and 6 , where the size and location of the planet-induced perturbations clearly depend on the value of $\alpha$.

Detectability is determined not just by size of the perturbations, but also by their duration. The duration depends on the value of the orbital period and on the mass ratio. While shorter orbital periods increase detectability by increasing the number of repetitions, the faster motion associated with them decreases the duration of perturbations. On the other hand, increasing the mass ratio $q$, between the planet and star always increases the duration. Let $T_{\text {dev }}$ represent the duration of perturbations. Di Stefano (2012) shows that

$$
T_{\text {dev }}=2.5 \xi P_{\text {orb }} 10^{\left[0.5 \log _{10}(q)-0.2\right]}
$$

where the value of $\xi$ depends on the observing setup, and can be taken to be of order unity in this discussion. (See
Di Stefano 2012 for more details.) Figure 5 displays the early-time and late-time perturbations associated with close-orbit planets. It shows that like wide-orbit planets, close-orbit planets can produce deviations both in advance of and after the closest approach. The time at which the deviations start is the time at which the source star enters the region with magnification perturbations at $R_{\alpha}$. Let $t_{\max }$ be the time at which deviations related to the planet begin (before the time of closest passage) or end (after the time of closest passage), representing the maximum time difference between the time of closest approach and the time at which deviations occur. If we measure time in days we have the analogue to Equation (3) for close-orbit planets:

$$
\left|t_{\max }-t_{0}\right| \sim 100 \text { days }\left(\frac{0.01 \frac{\theta_{E}}{\text { day }}}{\mu}\right)\left(\left(\frac{1}{\alpha}-\alpha\right)^{2}-\beta^{2}\right)^{\frac{1}{2}}
$$

The smaller the value of $\alpha$, the larger the value of $R_{\alpha}$, and the longer the difference in time between the closest approach and the start of close-planet-lens deviations. This is shown in Figure 5, which plots the difference between the magnification which includes the effect of the planet, $A_{p l}$, and the point-lens magnification, $A_{p t}$, expected if there is no planet. Figure 5 illustrates that the effects diminish in size as the value of $\alpha$ becomes smaller. Thus the effects of a 3 -Jupiter-mass planet at $\alpha=0.15$ would be challenging for ground-based observations, while a Saturn-mass planet could easily be discerned for $\alpha$ larger than about 0.3. The early-time and late-time perturbations are also shown in the top two panels of Figure 6.

The bottom panel of Figure 6 illustrates that the deviations produced by close-orbit planets can be detected even when the distance of closest approach is larger. The example shown in this panel has $\beta=5$. It is interesting to note that $A_{p t}$ reaches a maximum value of only 1.00275 . Thus, if we had plotted only the raw magnification, $A_{p l}$, the light curve would be essentially the same as the one shown. Thus, the signature of the planet would be an oscillatory deviation from baseline, of finite duration.

Another similarity with the wide-orbit case is that we can compute a specific value of $\alpha$ for a planet whose lensing signature we are capable of detecting (given the sensitivity and cadence of sampling) on a given day. Thus, when we do not find evidence of planets, we can place quantifiable limits on their existence.

Note that, by selecting VB 10 as an example, we have chosen a star with particularly high proper motion. Such stars are the most likely to produce events that can be predicted. The high proper motion does decrease the time duration of deviations, which makes detection more challenging. Other cases are shown in Di Stefano (2012) and in a future paper, based on Di Stefano, Matthews, \& Lépine (2012).

In addition to the repeating small deviations in the wings of the light curves, Figure 5 and the top two panels of Figure 6 show another interesting effect. This is that, for small values of $\beta$, there is a second region displaying significant perturbations. This region is near the light curve's peak, when the background star makes its closest approach to the foreground star. It corresponds to the reflexive motion of the central star and is larger for larger 


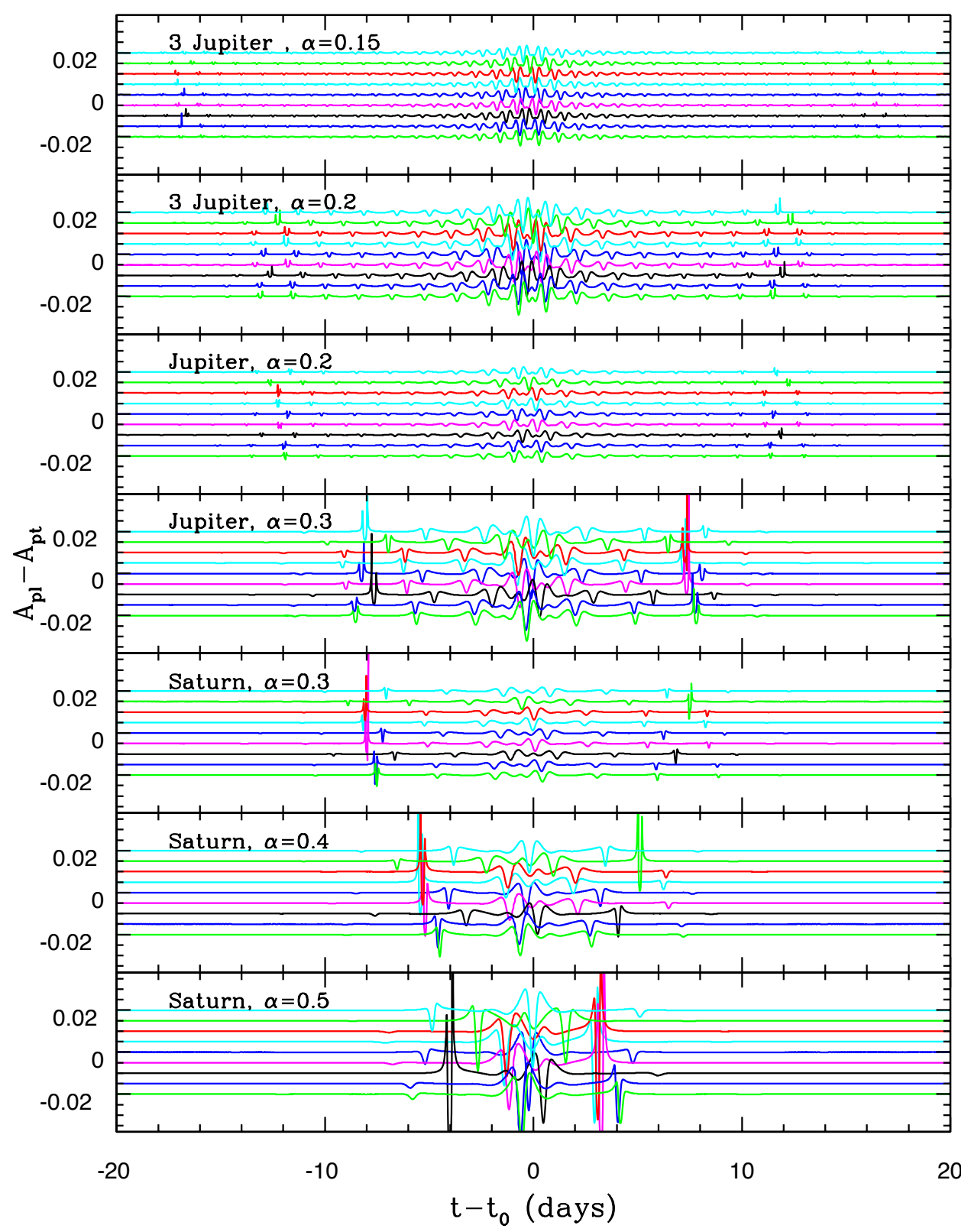

Figure 5. The difference between planet-lens $\left(A_{p l}\right)$ and point-lens $\left(A_{p t}\right)$ behavior as a function of $t-t_{0}$ for close-orbit planets. This illustrates planet effects for a range of orbital separations $(\alpha)$ and masses on the 5 mas approach $(\beta=0.5)$. Light curves from different initial orbital phases are shown with vertical offsets for clarity. Small, quasiperiodic deviations from single lens behaviour are observed, and there is increased activity in the region of $u \sim 1 / \alpha-\alpha$, near the beginning and end of the variability pattern. The deviations close to $t_{0}$ are caused by reflexive motion of the central star.

orbits. The size of the reflexive effects also increases with the mass of the close-orbit planet.

Just as there is a largest orbital separation for a wideorbit planet, there is a smallest orbital separation for a close-orbit planet. To avoid catastrophic tidal disruption a planet must satisfy the condition $r_{h} \gtrsim 2 r_{p}$, where $r_{h}$ is the Hill radius given by $r_{h} \approx a\left(\frac{m_{p}}{3 M_{*}}\right)^{1 / 3}$. Thus the minimum orbital separation of a planet around VB 10 is roughly given by

$$
a_{\text {min }} \sim 0.3 R_{\odot} \frac{r_{p}}{r_{J}}\left(\frac{M_{*}}{0.075 M_{\odot}}\right)^{1 / 3}\left(\frac{0.001 M_{\odot}}{m_{p}}\right)^{1 / 3}
$$

This corresponds to $\alpha_{\text {min }} \sim 0.03$ for the VB 10 case. The deviations produced by a planet in such a close orbit would occur when the position of the source star on the sky was approximately $30 R_{E}$ from the position of the foreground star. Their magnitude would be so small that they would be difficult to detect with today's technology. Event Probabilities: In Figures 5 and 6 , each panel contains 9 independent light curves, slightly displaced from each other so that the variations in each can be resolved. The nine light curves in each panel differ from each other only in that a different initial phase was chosen for each. The effects were similar in all cases. Had the perturbations been potentially detectable for only a small fraction of initial phases, then the probability of planet detection would be comparable to the value of that fraction. The ubiquity of detectable results indicates that, if the observational strategy is capable of detecting 


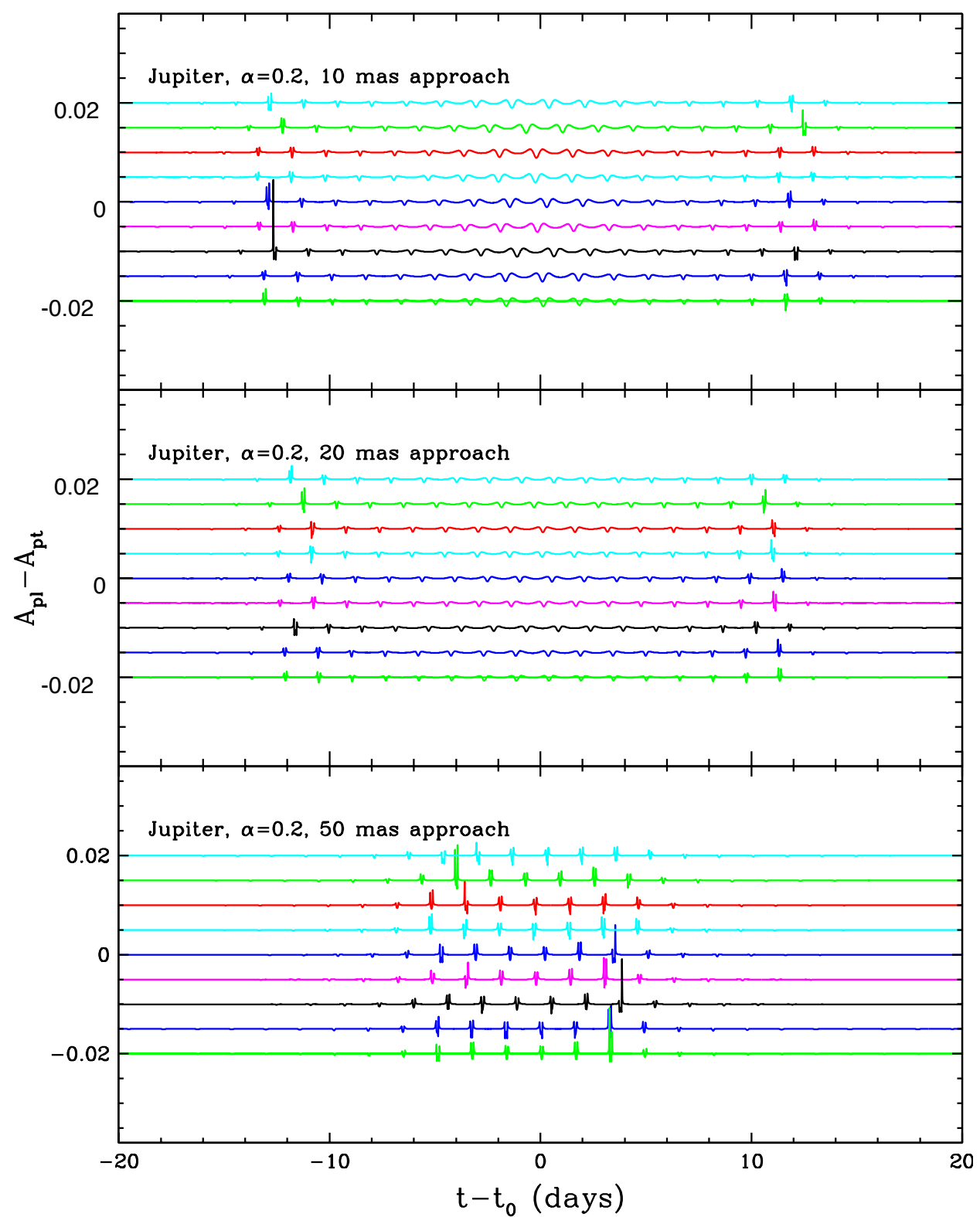

Figure 6. The difference between planet-lens $\left(A_{p l}\right)$ and point-lens $\left(A_{p t}\right)$ behavior as a function of $t-t_{0}$, analogously to Figure 4 . This illustrates close-orbit planet effects for $\alpha=0.2$, for a Jupiter-mass planet and $b=5,10,20$ mas $(\beta=1,2,5)$. The light curves from different phases are plotted with vertical offsets for clarity so the y axis should be seen as a relative scale only. Small, quasiperiodic deviations from single lens behaviour are produce, and there is increased activity in the region of $u \sim 1 / \alpha-\alpha$, near the beginning and end of the periodic sequence. The deviations close to $t_{0}$ are caused by reflexive motion of the central star.

deviations caused by planets with a given value of $\alpha$, orbital period, and $q$, then it will detect such planets with certainty. Thus, either close-orbit planets will be discovered, or limits on their existence can always be placed, if the observing conditions are favorable and the monitoring program is well designed. The values of $\alpha$ for which we can derive limits or else guarantee a detection are determined by the photometric sensitivity, whilst those of $q$ are determined by the cadence of sampling.

\section{3. "Resonant" Regime}

When the possibility for discovering planets via microlensing was first discussed (Mao \& Paczyński 1991; Gould \& Loeb 1992), attention focused on passages of the source star near or behind caustic structures like those shown in Figure 2. The associated light curves can show dramatic effects, although these can be moderated by the smoothing effect associated with the finite size of the source. Until now, microlensing searches for planets have focused on finding planets in the resonant zone. Lensing events are found by observing teams monitoring wide fields. Events judged to have a high probability of exhibiting planet-lens signatures are targeted for almost continuous monitoring during an interval (which can be as short as hours) when the magnification is deemed likely to be significantly influenced by the presence of planets. The 16 planets discovered via lensing have been found through such methods and are squarely in or near the borders of the zone for resonant lensing.

Light Curves: The associated light curves are therefore familiar from both calculations and observations. The new element introduced when the lens is a nearby fore- 


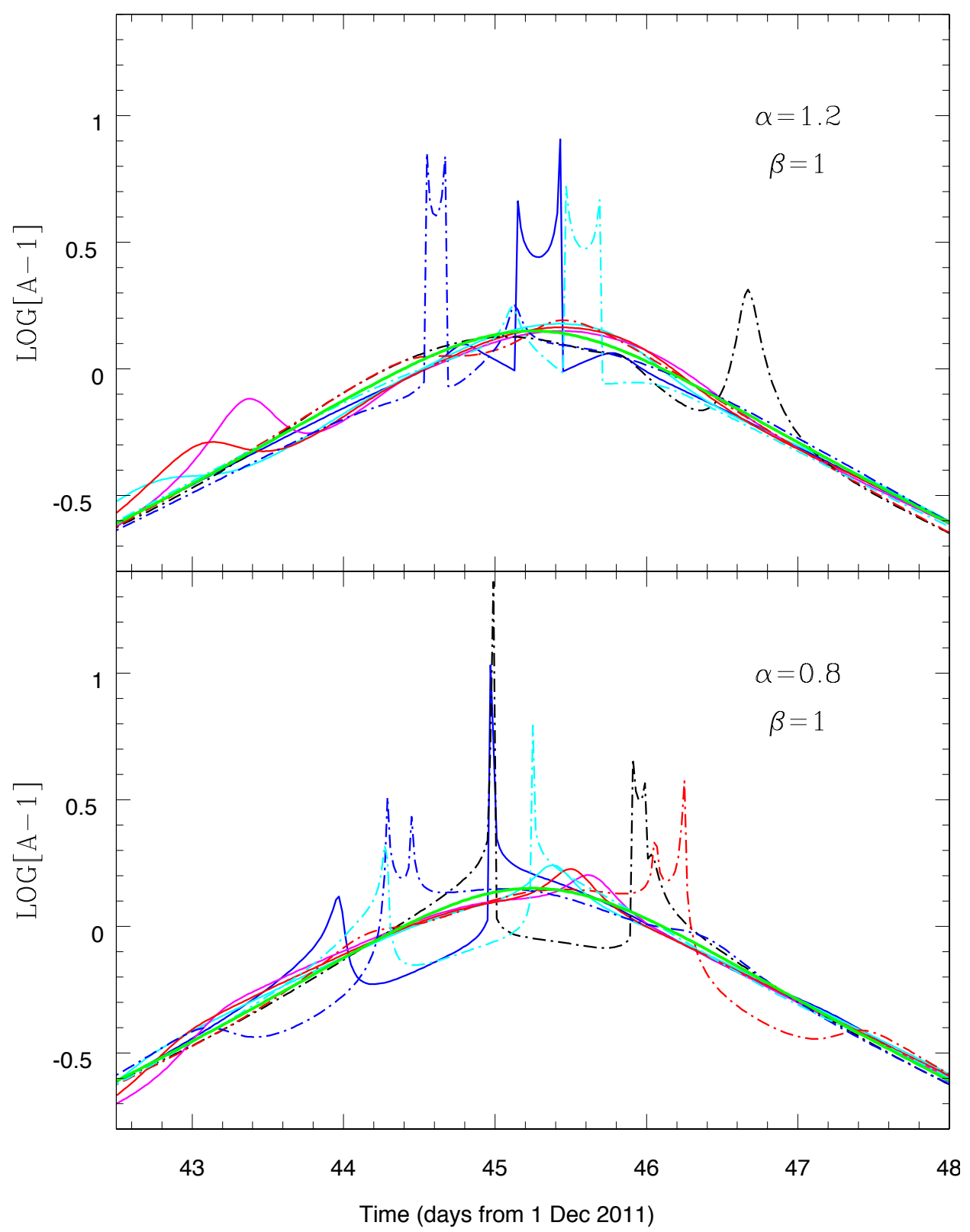

Figure 7. Simultaneously plotted light curves for a range of randomly sampled initial phases (in different colours), $\phi_{0}$, for two different values of $\alpha$ on a path that approaches within one Einstein radius of the central star. These light curves illustrate the types of features associated with lensing in the resonant zone. Caustic crossings can cause very sharp increases in magnification that are easily detectable, but the smoother curves demonstrate that significant deviations can occur even when caustics are not crossed. Ten separate light curves are plotted in each panel. The fact that a large fraction of them exhibit high magnifications and/or significant deviations from the point-lens form shows that the probability of detectable planet-lens distortions is large.

ground star is that the orbital size for values of $\alpha$ in the resonant zone can be small enough to make the orbital periods relatively short. Thus, rotation can play a significant role in determining the form of the light curves. This is illustrated in Figure 7. Because of the effect of orbital motion, the individual light curves plotted there exhibit more structure than typical for more distant lenses with planets in the zone for resonant lensing.

Event Probabilities: The closer the approach, the higher the probability of detecting events in which the source has passed near a caustic structure. This is illustrated in Figure 7, where light curves for a 5 mas approach of VB 10 to a background star are shown. For $\alpha=1.2$ (top panel) and $\alpha=0.8$ (bottom panel), ten ran- domly selected initial phases were each used to generate a light curve. For $\alpha=1.2$, eight of the light curves showed significant deviations, three involving caustic crossings (those exhibiting wall-like rises and falls). For $\alpha=0.8$, all ten light curves displayed significant variations, with an even larger number of caustic crossings than for $\alpha=1.2$. This illustrates that, for close passages between a background star and a nearby planetary system, the probability of detecting any planets inhabiting the zone for resonant lensing is close to unity. 


\section{CONCLUSIONS}

We have studied, for the first time, the characteristics and timing of planet-influenced events that can occur when a nearby foreground star makes a predicted close approach to a background star. Perhaps the most interesting finding is that the probability of discovering planets can be high enough to make monitoring worthwhile even when the passage between the foreground and background star is not that close. This is because the Einstein rings of nearby lenses can be large (from around a few mas to 30 - 40 mas), while close orbit planets, should they exist, are almost certain to produce detectable events for approaches of $(5-6) \theta_{E}$ or closer. These events could occur weeks or months before (or after) the time of closest approach. In addition, wide-orbit planets can produce events for even more distant approaches, and at even earlier or later times, although with smaller probability. The detection of planets in the resonant zone requires the closest approaches and is therefore most likely just near the time of closest approach, during an interval when the magnification reaches its peak value.

In order to relate the type of event to the time at which it is expected to occur (relative to the time of closest approach), we have introduced the notion of detection regimes, the regions within which it is most likely to detect planets in each zone. The close-orbit detection regime is the region through which the source must pass in order for us to detect close-orbit planets $(\alpha<0.5)$. The regime contains the zone where the planets are located, but also extends well beyond it. The resonant regime contains a disk encompassing the central star, as well as the annulus that constitutes the zone for resonant lensing $(0.5<\alpha<2)$. The wide regime, encompasses the entire lens plane, from the central star out to the radius of the farthest planet. Wide orbit planets can be detected at very early and/or at very late times and for distant approaches; the light curves are similar to the light curves expected for low-mass isolated lenses. In addition, if the approach is close enough to generate a stellar-lens event, the reflexive motion caused by a distant planet can be magnified, influencing the light curve shape, even if the wide-orbit planet does not itself generate an event. Close-orbit planets can be detected at intermediate times and for approaches less than about $6 \theta_{E}$. The light curve signatures are characteristic up-down-up-down features whose duration and baseline magnification can be used to determine the characteristics of the planet and its orbit. Resonant-orbit planets can be detected only during close approaches. When the lens is nearby enough for a close passage to be predicted, orbital motion can increase the structure in the light curve and thus increase the probability of planet detection.

These theoretical results provide a good basis for the design of observing programs that can effectively take advantage of close passages between nearby high-propermotion stars and background stars. In a future paper, based on DiStefano, Matthews, \& Lépine (2012), we specifically address the design of optimal programs, with the goal of opening up a new and productive approach to the discovery and study of nearby planets.

\section{Acknowledgements}

We thank Christopher Stubbs; Christopher Crockett; Fred Walters; David Charbonneau, Zachory Berta and the MEarth project; Jochen Greiner at GROND; Matthew Templeton and the AAVSO for their help and advice. This work was supported in part by NSF under AST-0908878 and under AST-0908406.

\section{REFERENCES}

Aihara, H., Allende Prieto, C., An, D., et al. 2011, ApJS, 193, 29

Batista, V., et al. 2011, A\&A, 529, 102

Bond, I. A., Abe, F., Dodd, R. J., et al. 2001, MNRAS, 327, 868

Di Stefano, R. 1999, ApJ, 512, 558

Di Stefano, R., 2008a, ApJ, 684, 46

Di Stefano, R., 2012, ApJ, 752, 105

Di Stefano, R., 2008b, ApJ, 684, 59

Di Stefano, R., Matthews, J., Lépine, S. 2012, arXiv:1202.5314

Di Stefano, R., \& Night, C. 2008, arXiv:0801.1510

Di Stefano, R., \& Scalzo, R. A. 1999a, ApJ, 512, 564

Di Stefano, R., \& Scalzo, R. A. 1999b, ApJ, 512, 579

Dominik, M., \& Sahu, K. C. 2000, ApJ, 534, 213

Einstein, A. 1936, Science, 84, 506

Guo, X., et al. 2011, arXiv:1112.4608G

Gould, A., \& Loeb, A. 1992, ApJ, 396, 104

Griest, K., \& Safizadeh, N. 1998, ApJ, 500, 37

Kaiser, N. 2004, SPIE, 5489, 11

Lépine, S., \& DiStefano, R. 2012, ApJ, 749, L6

Lépine, S., \& Gaidos, E. 2011, AJ, 142, 138

Lépine, S., \& Shara, M. M. 2005, AJ, 129, 1483

Mao, S., \& Paczynski, B. 1991, ApJ, 374, 37

Udalski, A. 2003, AcA, 53, 291

Wambsganss, J. 1997, MNRAS, 284, 172 